\title{
Catalan and Motzkin Integral Representations
}

\author{
Peter McCalla and Asamoah Nkwanta \\ Department of Mathematics \\ Morgan State University \\ Baltimore, MD 21251 \\ USA \\ peter.mccalla@morgan.edu \\ asamoah.nkwanta@morgan.edu
}

\begin{abstract}
We present new proofs of eight integral representations of the Catalan numbers. Then, we create analogous integral representations of the Motzkin numbers and obtain new results. Most integral representations of counting sequences found in the literature are proved by using advanced mathematical techniques. All integral representations in this paper are proved by using standard techniques from integral calculus. Thus, we provide a more simplistic approach of proving integral representations of the Catalan and Motzkin numbers.
\end{abstract}

\section{Introduction}

The Catalan numbers A000108 [11] are a sequence of natural numbers that are defined as

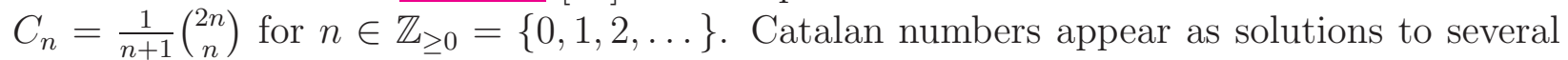
combinatorics problems. See Stanley $[12,13,14]$ for a number of combinatorial and analytical interpretations of $C_{n}$. It is closely related to the Motzkin numbers $M_{n} \underline{\text { A001006 [11] defined }}$ as [1]

$$
M_{n}=\sum_{k=0}^{\lfloor n / 2\rfloor}\left(\begin{array}{c}
n \\
2 k
\end{array}\right) C_{k} .
$$

Finding integral representations of counting numbers is a topic of interest for its intrinsic value. Various integral representations of $C_{n}$ are given in the literature, derived from methods involving Mellin transforms, Chebyshev polynomials, the Cauchy integral formula, and other advanced techniques that are beyond integral calculus. In this paper, we present several integral representations of $C_{n}$, then using two new results, prove analogous integral presentations of $M_{n}$. All representations are proved by using standard techniques from integral calculus. However, the authors do not claim that this paper contains all known integral representations of $C_{n}$ and $M_{n}$. 
Section 2 surveys the Catalan representations and Section 3 presents the Motzkin representations. What is new in Section 2 are the proofs. Section 3 contains new results: Theorems 10 and 11 and Corollaries 12 and 13. The only known integral representations of $M_{n}$ in Section 3 are Corollary 12(e) and (f). They are listed in OEIS [11] and there are no references to proofs. The proofs of Corollary $12(\mathrm{e})$ and (f) are also new.

\section{Integral representations of $C_{n}$}

We list eight integral representations of $C_{n}$. The first representation will be proved directly and subsequent representations will be proved using equivalence. We first prove the following lemma.

Lemma 1. For $r, s \in \mathbb{Z}_{\geq 0}$ and positive real number $a$,

$$
\int_{0}^{a / 2} \cos ^{r}\left(\frac{\pi x}{a}\right) \sin ^{s}\left(\frac{\pi x}{a}\right) d x=(-1)^{r} \int_{a / 2}^{a} \cos ^{r}\left(\frac{\pi x}{a}\right) \sin ^{s}\left(\frac{\pi x}{a}\right) d x .
$$

Proof. Using the integral identity $\int_{0}^{A} f(x) d x=\int_{0}^{A} f(A-x) d x$ for continuous function $f$ and the subtraction trigonometric identities $\sin (M-N)=\sin M \cos N-\cos M \sin N$ and $\cos (M-N)=\cos M \cos N+\sin M \sin N$ gives

$$
\begin{aligned}
\int_{0}^{a / 2} \cos ^{r}\left(\frac{\pi x}{a}\right) \sin ^{s}\left(\frac{\pi x}{a}\right) d x & =\int_{0}^{a / 2} \cos ^{r}\left(\frac{\pi}{2}-\frac{\pi x}{a}\right) \sin ^{s}\left(\frac{\pi}{2}-\frac{\pi x}{a}\right) d x \\
& =\int_{0}^{a / 2} \sin ^{r}\left(\frac{\pi x}{a}\right) \cos ^{s}\left(\frac{\pi x}{a}\right) d x
\end{aligned}
$$

Let $u=x+\frac{a}{2}$. Then by using the identities $\sin \left(M-\frac{\pi}{2}\right)=-\cos M$ and $\cos \left(M-\frac{\pi}{2}\right)=$ $\sin M$,

$$
\begin{aligned}
\int_{0}^{a / 2} \sin ^{r}\left(\frac{\pi x}{a}\right) \cos ^{s}\left(\frac{\pi x}{a}\right) d x & =\int_{a / 2}^{a} \sin ^{r}\left(\frac{\pi u}{a}-\frac{\pi}{2}\right) \cos ^{s}\left(\frac{\pi u}{a}-\frac{\pi}{2}\right) d u \\
& =(-1)^{r} \int_{a / 2}^{a} \cos ^{r}\left(\frac{\pi u}{a}\right) \sin ^{s}\left(\frac{\pi u}{a}\right) d u
\end{aligned}
$$

Theorem 2. ([7]) For $n \in \mathbb{Z}_{\geq 0}$,

$$
C_{n}=\frac{4^{n}}{(n+1) \pi} \int_{-1}^{1} \frac{x^{2 n}}{\sqrt{1-x^{2}}} d x .
$$

Proof. We start with the identity [5, 15]

$$
\left(\begin{array}{c}
2 n \\
n
\end{array}\right)=\frac{2 \cdot 4^{n}}{\pi} \int_{0}^{\pi / 2} \cos ^{2 n} x d x
$$


Using Lemma 1 with $r=2 n, s=0$, and $a=\pi$,

$$
\left(\begin{array}{c}
2 n \\
n
\end{array}\right)=\frac{4^{n}}{\pi} \int_{0}^{\pi} \cos ^{2 n} x d x
$$

Using the substitution $u=\cos x$, we get $d x=-\frac{1}{\sqrt{1-u^{2}}} d u$ and

$$
\left(\begin{array}{c}
2 n \\
n
\end{array}\right)=\frac{4^{n}}{\pi} \int_{-1}^{1} \frac{u^{2 n}}{\sqrt{1-u^{2}}} d u \text {. }
$$

The result follows by multiplying both sides by $\frac{1}{n+1}$.

Theorem 3. ([7]) For $n \in \mathbb{Z}_{\geq 0}$,

$$
C_{n}=\frac{4^{n}}{(n+1) \pi} \int_{0}^{1} \frac{x^{n}}{\sqrt{x-x^{2}}} d x .
$$

Proof. The integrand in (2) is an even function, so

$$
\begin{aligned}
\frac{4^{n}}{(n+1) \pi} \int_{-1}^{1} \frac{x^{2 n}}{\sqrt{1-x^{2}}} d x & =\frac{4^{n}}{(n+1) \pi} \int_{0}^{1} \frac{2 x^{2 n}}{\sqrt{1-x^{2}}} d x \\
& =\frac{4^{n}}{(n+1) \pi} \int_{0}^{1} \frac{x^{2 n}}{x \sqrt{1-x^{2}}} 2 x d x \\
& =\frac{4^{n}}{(n+1) \pi} \int_{0}^{1} \frac{\left(x^{2}\right)^{n}}{\sqrt{\left(x^{2}\right)-\left(x^{2}\right)^{2}}} d\left(x^{2}\right) \\
& =\frac{4^{n}}{(n+1) \pi} \int_{0}^{1} \frac{x^{n}}{\sqrt{x-x^{2}}} d x .
\end{aligned}
$$

Theorem 4. ([8, 9]) For $n \in \mathbb{Z}_{\geq 0}$,

$$
C_{n}=\frac{1}{2 \pi} \int_{0}^{4} x^{n} \sqrt{\frac{4-x}{x}} d x .
$$

Proof. From (4), let $y=4 x$. Then, we have

$$
\begin{aligned}
\frac{4^{n}}{(n+1) \pi} \int_{0}^{1} \frac{x^{n}}{\sqrt{x-x^{2}}} d x & =\frac{4^{n}}{(n+1) \pi} \int_{0}^{4} \frac{\left(\frac{y}{4}\right)^{n}}{\sqrt{\frac{y}{4}-\frac{y^{2}}{16}}} \frac{1}{4} d y \\
& =\frac{1}{(n+1) \pi} \int_{0}^{4} \frac{y^{n}}{\sqrt{\frac{4 y-y^{2}}{16}}} \frac{1}{4} d y \\
& =\frac{1}{(n+1) \pi} \int_{0}^{4} \frac{y^{n}}{\sqrt{4 y-y^{2}}} d y \\
& =\frac{1}{(n+1) \pi} \int_{0}^{4} \frac{y^{n+1}}{y^{2} \sqrt{\frac{4-y}{y}}} d y .
\end{aligned}
$$


Using integration by parts with $u=y^{n+1}$ and $d v=\frac{1}{y^{2} \sqrt{\frac{4-y}{y}}} d y$,

$$
\begin{aligned}
\frac{1}{(n+1) \pi} \int_{0}^{4} \frac{y^{n+1}}{y^{2} \sqrt{\frac{4-y}{y}}} d y & =\frac{1}{(n+1) \pi}\left(\frac{n+1}{2} \int_{0}^{4} y^{n} \sqrt{\frac{4-y}{y}} d y\right) \\
& =\frac{1}{2 \pi} \int_{0}^{4} y^{n} \sqrt{\frac{4-y}{y}} d y .
\end{aligned}
$$

Theorem 5. ([9]) For $n \in \mathbb{Z}_{\geq 0}$,

$$
C_{n}=\frac{2^{2 n+2}}{\pi} \int_{0}^{\infty} \frac{x^{2}}{\left(1+x^{2}\right)^{n+2}} d x .
$$

Proof. Starting from (5), use the reverse substitution $x=\frac{4}{1+t^{2}}$. Then,

$$
\begin{aligned}
\frac{1}{2 \pi} \int_{0}^{4} x^{n} \sqrt{\frac{4-x}{x}} d x & =\frac{1}{2 \pi} \int_{0}^{\infty}\left(\frac{4}{1+t^{2}}\right)^{n} \frac{8 t^{2}}{\left(1+t^{2}\right)^{2}} d t \\
& =\frac{4^{n} \cdot 8}{2 \pi} \int_{0}^{\infty} \frac{t^{2}}{\left(1+t^{2}\right)^{n+2}} d t \\
& =\frac{2^{2 n+2}}{\pi} \int_{0}^{\infty} \frac{t^{2}}{\left(1+t^{2}\right)^{n+2}} d t .
\end{aligned}
$$

Theorem 6. ([16]) For $n \in \mathbb{Z}_{\geq 0}$,

$$
C_{n}=\int_{0}^{1}(2 \cos (\pi x))^{2 n}\left(2 \sin ^{2}(\pi x)\right) d x .
$$

Proof. From (6), we use a substitution that is useful for integrating rational functions involving sine and cosine - the Weierstrass substitution $x=\tan \left(\frac{\pi t}{2}\right)$. By constructing a right triangle with angle $\frac{\pi t}{2}$, we get $\cos \left(\frac{\pi t}{2}\right)=\frac{1}{\sqrt{1+x^{2}}}$ and $\sin \left(\frac{\pi t}{2}\right)=\frac{x}{\sqrt{1+x^{2}}}$. Then

$$
\frac{2^{2 n+2}}{\pi} \int_{0}^{\infty} \frac{x^{2}}{\left(1+x^{2}\right)^{n+2}} d x=2^{2 n+1} \int_{0}^{1} \cos ^{2 n}\left(\frac{\pi t}{2}\right) \sin ^{2}\left(\frac{\pi t}{2}\right) d t
$$

Let $u=\frac{t}{2}$. Then,

$$
\begin{aligned}
2^{2 n+1} \int_{0}^{1} \cos ^{2 n}\left(\frac{\pi t}{2}\right) \sin ^{2}\left(\frac{\pi t}{2}\right) d t & =2^{2 n+2} \int_{0}^{1 / 2} \cos ^{2 n}(\pi u) \sin ^{2}(\pi u) d u \\
& =2^{2 n+1} \int_{0}^{1} \cos ^{2 n}(\pi u) \sin ^{2}(\pi u) d u
\end{aligned}
$$

where the last equality is a consequence of Lemma 1. 
Theorem 7. $([2,6,9])$ For $n \in \mathbb{Z}_{\geq 0}$,

$$
C_{n}=\frac{2^{2 n+5}}{\pi} \int_{0}^{1} \frac{x^{2}\left(1-x^{2}\right)^{2 n}}{\left(1+x^{2}\right)^{2 n+3}} d x .
$$

Proof. Use Lemma 1 to change (7) back to

$$
2^{2 n+2} \int_{0}^{1 / 2} \cos ^{2 n}(\pi x) \sin ^{2}(\pi x) d x
$$

Let $u=\tan \left(\frac{\pi x}{2}\right)$. Then, $\cos (\pi x)=\frac{1-u^{2}}{1+u^{2}}, \sin (\pi x)=\frac{2 u}{1+u^{2}}, d x=\frac{2}{\pi} \frac{d u}{1+u^{2}}$, and

$$
\begin{aligned}
2^{2 n+2} \int_{0}^{1 / 2} \cos ^{2 n}(\pi x) \sin ^{2}(\pi x) d x & =\frac{2^{2 n+3}}{\pi} \int_{0}^{1}\left(\frac{1-u^{2}}{1+u^{2}}\right)^{2 n}\left(\frac{2 u}{1+u^{2}}\right)^{2} \frac{1}{1+u^{2}} d u \\
& =\frac{2^{2 n+5}}{\pi} \int_{0}^{1} \frac{u^{2}\left(1-u^{2}\right)^{2 n}}{\left(1+u^{2}\right)^{2 n+3}} d u .
\end{aligned}
$$

Theorem 8. ([2, 9]) For $n \in \mathbb{Z}_{\geq 0}$,

$$
C_{n}=\frac{2^{2 n+1}}{\pi} \int_{-1}^{1} x^{2 n} \sqrt{1-x^{2}} d x .
$$

Proof. From (8), use the reverse substitution $x=\sqrt{\frac{1-u}{1+u}}$ to get

$$
\begin{aligned}
\frac{2^{2 n+5}}{\pi} \int_{0}^{1} \frac{x^{2}\left(1-x^{2}\right)^{2 n}}{\left(1+x^{2}\right)^{2 n+3}} d x & =\frac{2^{2 n+5}}{\pi} \int_{1}^{0} \frac{\frac{1-u}{1+u}\left(1-\frac{1-u}{1+u}\right)^{2 n}}{\left(1+\frac{1-u}{1+u}\right)^{2 n+3}} \frac{-1}{(1+u) \sqrt{1-u^{2}}} d u \\
& =\frac{2^{2 n+5}}{\pi} \int_{0}^{1} \frac{\frac{1-u}{1+u}\left(\frac{2 u}{1+u}\right)^{2 n}}{\left(\frac{2}{1+u}\right)^{2 n+3}(1+u) \sqrt{1-u^{2}}} d u \\
& =\frac{2^{2 n+2}}{\pi} \int_{0}^{1} \frac{u^{2 n}(1-u)(1+u)}{\sqrt{1-u^{2}}} d u \\
& =\frac{2^{2 n+2}}{\pi} \int_{0}^{1} u^{2 n} \sqrt{1-u^{2}} d u .
\end{aligned}
$$

Since the integrand is an even function, the result follows.

Remark. This integral representation can be proved using the beta and gamma functions and related identities [9].

Theorem 9. ([9]) For $n \in \mathbb{Z}_{\geq 0}$,

$$
C_{n}=\frac{1}{2 \pi} \int_{-2}^{2} x^{2 n} \sqrt{4-x^{2}} d x
$$


Proof. From (9), let $u=2 x$. Then

$$
\begin{aligned}
\frac{2^{2 n+1}}{\pi} \int_{-1}^{1} x^{2 n} \sqrt{1-x^{2}} d x & =\frac{2^{2 n+1}}{\pi} \int_{-2}^{2}\left(\frac{u}{2}\right)^{2 n} \sqrt{1-\left(\frac{u}{2}\right)^{2}} \frac{d u}{2} \\
& =\frac{1}{\pi} \int_{-2}^{2} u^{2 n} \sqrt{1-\frac{u^{2}}{4}} d u \\
& =\frac{1}{2 \pi} \int_{-2}^{2} u^{2 n} \sqrt{4-u^{2}} d u
\end{aligned}
$$

\section{Integral representations of $M_{n}$}

The next two theorems will provide a link between the representations (2) and (4) - (10) and Corollaries 12 and 13.

Theorem 10. Let $n \in \mathbb{Z}_{\geq 0}$ and $a, b \in \mathbb{R} \cup\{ \pm \infty\}$. Suppose $C_{n}$ can be written in the form

$$
C_{n}=\int_{a}^{b}(f(x))^{2 n} g(x) d x
$$

for integrable functions $f$ and $g$. Then

$$
M_{n}=\frac{1}{2} \int_{a}^{b}\left((1+f(x))^{n}+(1-f(x))^{n}\right) g(x) d x .
$$

Proof. Using (1) and the Binomial Theorem, we have

$$
\begin{aligned}
M_{n} & =\int_{a}^{b} \sum_{k=0}^{\lfloor n / 2\rfloor}\left(\begin{array}{c}
n \\
2 k
\end{array}\right)(f(x))^{2 k} g(x) d x \\
& =\frac{1}{2} \int_{a}^{b}\left(\sum_{k=0}^{n}\left(\begin{array}{l}
n \\
k
\end{array}\right)(f(x))^{k}+\sum_{k=0}^{n}\left(\begin{array}{l}
n \\
k
\end{array}\right)(-1)^{k}(f(x))^{k}\right) g(x) d x \\
& =\frac{1}{2} \int_{a}^{b}\left((1+f(x))^{n}+(1-f(x))^{n}\right) g(x) d x .
\end{aligned}
$$

Theorem 11. Let $n \in \mathbb{Z}_{\geq 0}$ and $a, b \in \mathbb{R} \cup\{ \pm \infty\}$. Suppose $C_{n}$ can be written in the form

$$
C_{n}=\frac{1}{n+1} \int_{a}^{b}(f(x))^{2 n} g(x) d x
$$

for integrable functions $f$ and $g$. Then

$$
M_{n}=\int_{a}^{b} \frac{\varphi_{n+2}(x)-\varphi_{n+1}(x)}{(f(x))^{2}} g(x) d x,
$$

where

$$
\varphi_{n}(x)=\frac{1}{n}\left((1+f(x))^{n}+(1-f(x))^{n}-2\right)
$$


Proof. We use the same techniques as the proof of Theorem 10; although, we need to initially convert $M_{n}$ to a double integral. From (1), we have

$$
\begin{gathered}
M_{n}=\int_{a}^{b} \sum_{k=0}^{\lfloor n / 2\rfloor}\left(\begin{array}{c}
n \\
2 k
\end{array}\right) \frac{1}{k+1}(f(x))^{2 k} g(x) d x \\
=\int_{a}^{b} \int_{0}^{1} \sum_{k=0}^{\lfloor n / 2\rfloor}\left(\begin{array}{c}
n \\
2 k
\end{array}\right)(f(x))^{2 k} y^{k} g(x) d y d x \\
=\int_{a}^{b} \int_{0}^{1} \sum_{k=0}^{\lfloor n / 2\rfloor}\left(\begin{array}{c}
n \\
2 k
\end{array}\right)(f(x) \sqrt{y})^{2 k} g(x) d y d x \\
=\frac{1}{2} \int_{a}^{b} g(x) \int_{0}^{1}\left(\sum_{k=0}^{n}\left(\begin{array}{c}
n \\
k
\end{array}\right)(f(x) \sqrt{y})^{k}+\sum_{k=0}^{n}\left(\begin{array}{c}
n \\
k
\end{array}\right)(-1)^{k}(f(x) \sqrt{y})^{k}\right) d y d x \\
=\frac{1}{2} \int_{a}^{b} g(x) \int_{0}^{1}\left((1+f(x) \sqrt{y})^{n}+(1-f(x) \sqrt{y})^{n}\right) d y d x \\
=\int_{a}^{b} g(x) \frac{1}{(f(x))^{2}}\left(\int_{1}^{1+f(x)}\left(u^{n+1}-u^{n}\right) d u+\int_{1}^{1-f(x)}\left(v^{n+1}-v^{n}\right) d v\right) d x,
\end{gathered}
$$

where $u=1+f(x) \sqrt{y}$ and $v=1-f(x) \sqrt{y}$. The result follows by evaluating the inner integrals and simplifying.

Corollary 12. For $n \in \mathbb{Z}_{\geq 0}$,

$$
\begin{aligned}
& \text { (a) } M_{n}=\frac{1}{4 \pi} \int_{0}^{4}\left((1+\sqrt{x})^{n}+(1-\sqrt{x})^{n}\right) \sqrt{\frac{4-x}{x}} d x, \\
& \text { (b) } M_{n}=\frac{2}{\pi} \int_{0}^{\infty}\left(\left(1+\frac{2}{\sqrt{1+x^{2}}}\right)^{n}+\left(1-\frac{2}{\sqrt{1+x^{2}}}\right)^{n}\right) \frac{x^{2}}{\left(1+x^{2}\right)^{2}} d x, \\
& \text { (c) } M_{n}=\int_{0}^{1}\left((1+2 \cos \pi x)^{n}+(1-2 \cos \pi x)^{n}\right) \sin ^{2} \pi x d x, \\
& \text { (d) } M_{n}=\frac{16}{\pi} \int_{0}^{1} \frac{x^{2}\left(\left(3-x^{2}\right)^{n}+\left(3 x^{2}-1\right)^{n}\right)}{\left(1+x^{2}\right)^{n+3}} d x, \\
& \text { (e) } M_{n}=\frac{2}{\pi} \int_{-1}^{1}(1+2 x)^{n} \sqrt{1-x^{2}} d x, \\
& \text { (f) } M_{n}=\frac{1}{2 \pi} \int_{-2}^{2}(1+x)^{n} \sqrt{4-x^{2}} d x .
\end{aligned}
$$

Remark. The integral representations in Corollary 12(e) and (f) are presented by Peter Lushny and Paul Barry, respectively, in OEIS [11]. 
Proof. (a) Use (11) on (5) with $f(x)=\sqrt{x}$ and $g(x)=\frac{1}{2 \pi} \sqrt{\frac{4-x}{x}}$.

(b) Use (11) on (6) with $f(x)=\frac{2}{\sqrt{1+x^{2}}}$ and $g(x)=\frac{4 x^{2}}{\pi\left(1+x^{2}\right)^{2}}$.

(c) Use (11) on (7) with $f(x)=2 \cos \pi x$ and $g(x)=2 \sin ^{2} \pi x$.

(d) Using (11) on (8) with $f(x)=\frac{2\left(1-x^{2}\right)}{1+x^{2}}$ and $g(x)=\frac{32 x^{2}}{\pi\left(1+x^{2}\right)^{3}}$, we get

$$
\begin{aligned}
M_{n} & =\frac{16}{\pi} \int_{0}^{1}\left(\left(1+\frac{2-2 x^{2}}{1+x^{2}}\right)^{n}+\left(1-\frac{2-2 x^{2}}{1+x^{2}}\right)^{n}\right) \frac{x^{2}}{\left(1+x^{2}\right)^{3}} d x \\
& =\frac{16}{\pi} \int_{0}^{1}\left(\left(\frac{3-x^{2}}{1+x^{2}}\right)^{n}+\left(\frac{3 x^{2}-1}{1+x^{2}}\right)^{n}\right) \frac{x^{2}}{\left(1+x^{2}\right)^{3}} d x \\
& =M_{n}=\frac{16}{\pi} \int_{0}^{1} \frac{x^{2}\left(\left(3-x^{2}\right)^{n}+\left(3 x^{2}-1\right)^{n}\right)}{\left(1+x^{2}\right)^{n+3}} d x .
\end{aligned}
$$

(e) Using (11) on (9) with $f(x)=2 x$ and $g(x)=\frac{2}{\pi} \sqrt{1-x^{2}}$, we get

$$
\begin{aligned}
M_{n} & =\frac{1}{\pi} \int_{-1}^{1}\left((1+2 x)^{n}+(1-2 x)^{n}\right) \sqrt{1-x^{2}} d x \\
& =\frac{1}{\pi}\left(\int_{-1}^{1}(1+2 x)^{n} \sqrt{1-x^{2}} d x+\int_{-1}^{1}(1-2 x)^{n} \sqrt{1-x^{2}} d x\right) .
\end{aligned}
$$

Perform the substitution $u=-x$ on the second integral to get the desired result.

(f) Using (11) on (10) with $f(x)=x$ and $g(x)=\frac{1}{2 \pi} \sqrt{4-x^{2}}$, we get

$$
\begin{aligned}
M_{n} & =\frac{1}{4 \pi} \int_{-2}^{2}\left((1+x)^{n}+(1-x)^{n}\right) \sqrt{4-x^{2}} d x \\
& =\frac{1}{4 \pi}\left(\int_{-2}^{2}(1+x)^{n} \sqrt{4-x^{2}} d x+\int_{-2}^{2}(1-x)^{n} \sqrt{4-x^{2}} d x\right) .
\end{aligned}
$$

Perform the substitution $u=-x$ on the second integral to get the desired result.

Alternative proof of Corollary 12(f): From the representation in (e), perform the substitution $u=2 x$ to get

$$
\begin{aligned}
\frac{1}{\pi} \int_{-2}^{2}(1+u)^{n} \sqrt{1-\frac{u^{2}}{4}} d u & =\frac{1}{\pi} \int_{-2}^{2}(1+u)^{n} \sqrt{\frac{4-u^{2}}{4}} d u \\
& =\frac{1}{2 \pi} \int_{-2}^{2}(1+u)^{n} \sqrt{4-u^{2}} d u
\end{aligned}
$$


Corollary 13. For $n \in \mathbb{Z}_{\geq 0}$,

(a) $M_{n}=\frac{1}{4 \pi} \int_{0}^{1} \frac{\phi_{n+2}(x)-\phi_{n+1}(x)}{x \sqrt{x-x^{2}}} d x$,

(b) $M_{n}=\frac{1}{2 \pi} \int_{-1}^{1} \frac{\psi_{n+2}(x)-\psi_{n+1}(x)}{x^{2} \sqrt{1-x^{2}}} d x$,

where

$$
\phi_{n}(x)=\frac{1}{n}\left((1+2 \sqrt{x})^{n}+(1-2 \sqrt{x})^{n}-2\right)
$$

and

$$
\psi_{n}(x)=\frac{1}{n}\left((1+2 x)^{n}-1\right) .
$$

Proof. (a) Use (12) on (4) with $f(x)=2 \sqrt{x}$ and $g(x)=\frac{1}{\pi \sqrt{x-x^{2}}}$.

(b) Use (12) on (2) with $f(x)=2 x$ and $g(x)=\frac{1}{\pi \sqrt{1-x^{2}}}$. The result follows by splitting the integral, then using the substitution $u=-x$ on the integrals with the expressions $(1-2 x)^{n+2}$ and $(1-2 x)^{n+1}$.

\section{Conclusion}

We give other known Catalan integral representations [7]:

$$
C_{n}=\frac{2^{2 n+1}}{(2 n+1) \pi} \int_{-1}^{1} \frac{x^{2 n+2}}{\sqrt{1-x^{2}}} d x
$$

and

$$
C_{n}=\frac{4^{n}}{n \pi} \int_{0}^{1} \frac{2 x^{n+1}-x^{n}}{\sqrt{x-x^{2}}} d x
$$

Also, from (3),

$$
C_{n}=\frac{4^{n}}{(n+1) \pi} \int_{0}^{\pi} \cos ^{2 n} x d x
$$

See Qi and Guo [9] for integral representations of $C_{n}$ and $\frac{1}{C_{n}}$.

We have presented several integral representations of $C_{n}$ and $M_{n}$. Finding integral representations of other counting numbers such as the Riordan numbers A005043, binomial coefficients of the Pascal triangle A109906, Fine numbers A000957, and RNA numbers $\underline{\text { A110320 }}$ [11] would be of interest. See Qi, Shi, and Guo [10] for interesting integral representations of the little A001003 and large A006318 Schröder numbers [11]; Dilcher [3], for the even Fibonacci numbers A014445 [11]; and Glasser and Zhou [4], for the Fibonacci numbers A000045 $[11]$.

Given the many combinatorial and analytic interpretations of $C_{n}$ and $M_{n}$, an important and interesting problem worth pursuing is to find related analytical interpretations of the integral representations. 


\section{Acknowledgment}

The authors would like to thank Rodney Kerby, Leon Woodson, and Guoping Zhang for useful comments on various drafts of the manuscript.

\section{References}

[1] F. Bernhart, Catalan, Motzkin, and Riordan numbers, Discrete Math. 204 (1999), 73112.

[2] T. Dana-Picard, Integral presentations of Catalan numbers and Wallis formula, Internat. J. Math. Ed. Sci. Tech. 42 (2011), 122-129.

[3] K. Dilcher, Hypergeometric functions and Fibonacci numbers, Fibonacci Quart. 38 (2000), 342-363.

[4] L. Glasser and Y. Zhou, An integral representation for the Fibonacci numbers and their generalization, Fibonacci Quart. 53 (2015), 313-318.

[5] T. Koshy, Catalan Numbers with Applications, Oxford University Press, 2009.

[6] A. Nkwanta and A. Tefera, Curious relations and identities involving the Catalan generating function and numbers, J. Integer Seq. 16 (2013), Article 13.9.5.

[7] A. Nkwanta and E. R. Barnes, Two Catalan-type Riordan arrays and their connections to the Chebyshev polynomials of the first kind, J. Integer Seq. 15 (2012), Article 12.3.3.

[8] K. A. Penson and J. M. Sixdeniers, Integral representations of Catalan and related numbers, J. Integer Seq. 4 (2001), Article 01.2.5.

[9] F. Qi and B. Guo, Integral representations of the Catalan numbers and their applications, Multidisciplinary Digital Publishing Institute 5 (2017).

[10] F. Qi, X. Shi, and B. Guo, Integral representations of the large and little Schröder numbers, Indian J. Pure Appl. Math. 49 (2018), 23-38.

[11] N. J. A. Sloane. The On-line Encyclopedia of Integer Sequences, published online at http://oeis.org, 2013.

[12] R. P. Stanley, Catalan Numbers, Cambridge University Press, 2009.

[13] R. P. Stanley, Enumerative Combinatorics, Vol. 2, Cambridge University Press, 1999.

[14] R. P. Stanley, Enumerative Combinatorics, Vol. 2, Catalan Addendum, published online at http://www-math.mit.edu/ rstan/ec/catadd.pdf, 2011. 
[15] J. Wiener, Integrals of $\cos ^{2 n} x$ and $\sin ^{2 n} x$, College Math. J. 31 (2000), 60-61.

[16] Q. Yuan, The Catalan numbers, regular languages, and orthogonal polynomials, 2009, https://qchu. wordpress. com/2009/06/07/.

Keywords: Catalan numbers, Motzkin numbers, integral representation, Weierstrass substitution.

(Concerned with sequences $\underline{\mathrm{A} 000045}, \underline{\mathrm{A} 000108}, \underline{\mathrm{A} 000957}, \underline{\mathrm{A} 001003}, \underline{\mathrm{A} 001006}, \underline{\mathrm{A} 005043}, \underline{\mathrm{A} 006318}$, $\underline{\mathrm{A} 014445}, \underline{\mathrm{A} 109906}$, and $\underline{\mathrm{A} 110320}$. 